\title{
Estudo anatômico, ultrassonográfico e tomográfico do aparato podotroclear de equinos adultos
}

[Anatomic, tomographic and sonographic study of podotrochlear apparatus of adult horses]

\author{
L.P. Souza ${ }^{1}$, Z. Bortolini ${ }^{2}$, T.R. Müller ${ }^{3}$, J.H. Fonteque ${ }^{3}$, J. Schade ${ }^{1}$, M.G. Luciani ${ }^{1}$, \\ D.S. Souza ${ }^{3}$, L.C. Vulcano ${ }^{2}$
}

\footnotetext{
${ }^{1}$ Aluno de pós-graduação - Universidade do Estado de Santa Catarina - Lages, SC

${ }^{2}$ Universidade Estadual Paulista "Júlio de Mesquita Filho" - Botucatu, SP

${ }^{3}$ Universidade do Estado de Santa Catarina - Lages, SC
}

\begin{abstract}
RESUMO
A síndrome do navicular é uma condição que envolve o aparato podotroclear e representa uma das causas mais comuns de claudicação dos membros torácicos de equinos. Portanto, o estudo complementar da região reveste-se de grande interesse quando se refere ao diagnóstico e tratamento das claudicações dos equinos. O objetivo deste estudo foi demonstrar as diferenças dos achados imagenológicos entre a ultrassonografia e a tomografia computadorizada na avaliação das estruturas palmares do aparato podotroclear de equinos adultos e hígidos, bem como a descrição das estruturas observadas nas imagens obtidas com essas técnicas. Para isso, foram realizadas imagens de quatro peças anatômicas, as quais foram posteriormente seccionadas e utilizadas para a descrição anatômica. A utilização de peças anatômicas auxilia no conhecimento da anatomia normal, o que leva à melhor interpretação das imagens e aumenta a especificidade diagnóstica na detecção das alterações que as doenças acarretam. A ultrassonografia fornece informações relevantes quanto às estruturas estudadas, e sua associação com a tomografia computadorizada aumentou a acurácia da investigação. Apesar de o uso da tomografia computadorizada ser mais indicada para tecido ósseo, ela fornece informações importantes, podendo ser usada como uma ferramenta útil quando não se tem disponível a ressonância magnética em razão do custo ou da disponibilidade.
\end{abstract}

Palavras-chave: diagnóstico, claudicação, navicular, tomografia computadorizada, ultrassom

\begin{abstract}
The navicular syndrome is a condition involving the podotrochlear apparatus and represents one of the most common causes of forelimb lameness in horses. Therefore, further study of this region is of interest when it comes to diagnosis and treatment of lameness in horses. The aim of this study was to demonstrate the differences between the imaging findings of ultrasonography and computed tomography in the evaluation of the palmar structures of the podotrochlear apparatus of healthy adult horses and description of the structures observed in images obtained with these techniques. For this, four images of four anatomical parts were performed, and subsequently sectioned and used for the anatomical description. The use of anatomical parts helps in the understanding of normal anatomy leading to a better interpretation of the images and increasing the specificity of the diagnostic for detecting changes that cause diseases. Ultrasonography provides relevant information about these structures to be studied and the association with computed tomography $(C T)$ increased the accuracy of the investigation. Despite the use of CT being more suitable for bone tissue it provides important information and can be used as a useful tool when there is no available MRI.
\end{abstract}

Keywords: diagnosis, lameness, navicular, computed tomography, ultrasound

Recebido em 15 de dezembro de 2016

Aceito em 19 de janeiro de 2017

E-mail: li.pasini.vet@gmail.com 


\section{INTRODUÇÃO}

A maioria das claudicações nos equinos envolve os membros torácicos e, destas, cerca de $95 \%$ originam-se distal ao carpo, envolvendo frequentemente o aparato podotroclear (Dyson e Marks, 2003; Stashak, 2005). Este é composto pelo osso sesamoide distal (navicular), por ligamentos sesamoides distal ímpar (LSDI) e colaterais, pelo tendão do músculo flexor digital profundo (TFDP) e pela bursa podotroclear (Busoni e Denoix, 2001). A alta incidência de afecções do aparato podotroclear nos membros torácicos deve-se principalmente ao fato de esses membros suportarem entre 60 e $65 \%$ do peso do animal, sendo sujeitos ao maior impacto contra o solo, o que é reduzido nos membros posteriores, pois atuam predominantemente como propulsores (Stashak, 2005; Dyson e Marks, 2003).

A afecção do aparato podotroclear é considerada uma desordem crônica e progressiva, que acomete equinos, principalmente atletas com mais de 10 anos de idade. É caracterizada por mudanças nas estruturas, incluindo a composição e função mecânica da cartilagem, do osso subcondral e dos tecidos moles adjacentes, como o TFDP, o LSDI, os ligamentos colaterais ou a bursa podotroclear (Turner, 1989; Wright, 1993; Stashak, 2005; Rijkenhuizen, 2006).

O diagnóstico da síndrome do navicular é comumente baseado no histórico, nos sinais clínicos, na resposta ao bloqueio anestésico dos nervos digitais palmares e na detecção de alterações pelo exame radiográfico (Turner, 1989), sendo este o método de imagem mais frequentemente utilizado (Busoni e Denoix, 2001). Entretanto, estudos revelam que o exame radiográfico fornece um detalhamento pobre devido à sobreposição de estruturas adjacentes e à pouca resolução espacial (Fio e Koblik, 1995). Além disso, em muitos casos, a síndrome do navicular pode ocorrer sem qualquer alteração radiográfica, sugerindo apenas o envolvimento dos tecidos moles adjacentes (Busoni e Denoix, 2001).

Com o auxílio de outras ferramentas imagenológicas e de novas técnicas de imagem associadas ao exame radiográfico, tem-se aprimorado o diagnóstico precoce das lesões que causam claudicação em equinos, em especial aquelas que envolvem a porção distal dos membros (Dyson e Marks, 2003; Stashak, 2005).

A avaliação ultrassonográfica da extremidade distal é uma modalidade de imagem que vem aprimorando os conhecimentos sobre as alterações que ocorrem no interior do casco (Rijkenhuizen, 2006). É amplamente utilizada em equinos e frequentemente a única opção diagnóstica em se tratando de claudicação relacionada a lesões na extremidade distal do membro (Whitcomb, 2009). Para a avaliação das estruturas intramurais, uma técnica útil para o diagnóstico em equinos que apresentam dor na região palmar do casco é a ultrassonografia transcuneal, pois se podem avaliar estruturas palmares relacionadas ao aparato podotroclear (Busoni et al., 2006).

A tomografia computadorizada é um método diagnóstico sofisticado, que obtém imagens seccionais de diversas partes do corpo, eliminando, dessa maneira, a sobreposição de estruturas. Essas imagens podem ser combinadas e manipuladas por programas de computador especiais para produzir imagens em diferentes planos anatômicos, até mesmo imagens tridimensionais, o que possibilita melhor avaliação das estruturas ósseas e articulares (Fio e Koblik, 1995; Rijkenhuizen, 2006). A tomografia computadorizada vem sendo utilizada para a investigação de lesões no interior do casco e de estruturas associadas que não são visibilizadas radiograficamente, sendo a melhor modalidade para detectar e acessar, em detalhes, alterações ósseas envolvendo o córtex e o trabeculado (Fio e Koblik, 1995; Whitton et al., 1998; Rijkenhuizen, 2006).

A avaliação do aparato podotroclear pela associação de dois métodos (ultrassonografia e tomografia computadorizada) reveste-se de grande importância para o diagnóstico e o tratamento da síndrome do navicular (Busoni e Denoix, 2001), porém os conhecimentos anatômico e imagenológico são de fundamental relevância para um diagnóstico mais fidedigno.

Dessa maneira, o presente estudo tem por objetivo demonstrar as diferenças entre as imagens obtidas pela ultrassonografia e pela tomografia computadorizada na avaliação do aparato podotroclear de equinos, bem como promover a descrição anatômica normal, 
a fim de aprimorar os conhecimentos anatomoimaginológicos dessa região e, assim, fornecer ao clínico e ao cirurgião de equinos mais opções diagnósticas, as quais, associadas aos exames clínico e radiográfico de rotina, podem fornecer um diagnóstico definitivo para claudicações relacionadas ao aparato podotroclear.

\section{MATERIAL E MÉTODOS}

Utilizou-se a porção distal de quatro peças anatômicas dos membros torácicos de equinos adultos, que foram a óbito por enfermidades não relacionadas com lesões no interior do estojo córneo. Essas peças foram mantidas em refrigeração até a realização dos exames de imagem e, em seguida, foram congeladas e seccionadas em plano sagital para avaliação macroscópica das estruturas referentes ao aparato podotroclear, descrição anatômica (Popesko, 1997; Denoix, 2000; Clayton e Flood, 2002; Budras e König, 2004) e comparação com os achados imagenológicos obtidos nos exames de ultrassonografia e tomografia computadorizada.

As imagens ultrassonográficas foram realizadas com o equipamento MyLab 70, da marca Esaote, utilizando-se transdutor linear multifrequencial (8-13MHz), em plano longitudinal, as quais foram armazenadas em hardware para posterior avaliação. Para a obtenção das imagens intramurais pela técnica de ultrassonografia transcuneal, o casco foi submergido em água morna por 15 a 30 minutos para o amolecimento da ranilha, que, posteriormente, foi aparada para melhorar acoplamento acústico. A escolha da frequência variou significativamente de acordo com as variações individuais do paciente, e a profundidade fixada em três a seis centímetros.

As imagens tomográficas foram realizadas com um aparelho de tomografia computadorizada helicoidal da marca Shimadzu, modelo SCT7800TC, com $120 \mathrm{kV}$ e $160 \mathrm{~mA}$, em cortes axiais contínuos de $1 \mathrm{~mm}$ de espessura de $1 \mathrm{~mm}$ de espaçamento desde a porção proximal da primeira falange até porção distal do casco. Todas as imagens foram obtidas em filtros para tecido ósseo e avaliadas em janela para tecido ósseo e tecidos moles, sendo armazenadas em hardware para posterior avaliação.

\section{RESULTADOS}

Para a investigação do aparato podotroclear pelos métodos de diagnóstico por imagem, fez-se necessário o estudo das estruturas presentes na região palmar do casco, sendo estas o osso navicular, o LSDI, o ligamento colateral (suspensor), o TFDP, o ligamento anular digital distal (LADD), o coxim digital e a bursa podotroclear. Essas estruturas podem ser observadas na face palmar da peça anatômica em corte sagital (Fig. 1) e comparadas com os achados imagenológicos obtidos pelos métodos de ultrassonografia (Fig. 2) e tomografia computadorizada (Fig. 3 e 4). Nenhuma lesão macroscópica foi identificada nas estruturas avaliadas, tanto nas peças quanto nas imagens ultrassonográficas e tomográficas.

O corte em plano sagital da peça anatômica (Fig. 1) demonstra as articulações interfalangeanas proximal e distal, bem como as estruturas da face palmar da região podotroclear. A articulação interfalangeana proximal articula a tróclea da falange proximal com a face articular proximal da falange média. $\mathrm{Na}$ articulação interfalangeana distal, articula-se a tróclea da falange média com as faces articulares da falange distal e do osso navicular, o qual se encontra na face palmar, mantendo constante o ângulo de inserção do TFDP. Sua cápsula articular forma evaginações nas faces dorsal e palmar, conhecidas como recessos. O recesso palmar esta localizado na região dorsal ao TFDP e é dividido em recesso palmar proximal e recesso palmar distal da articulação interfalangeana distal. Da margem distal do osso navicular até a margem palmar da superfície palmar da falange distal, localiza-se o LSDI. O ligamento sesamoide colateral (suspensor) está situado na borda proximal do osso navicular, sendo possível identificar apenas uma pequena porção desse ligamento no corte sagital. 


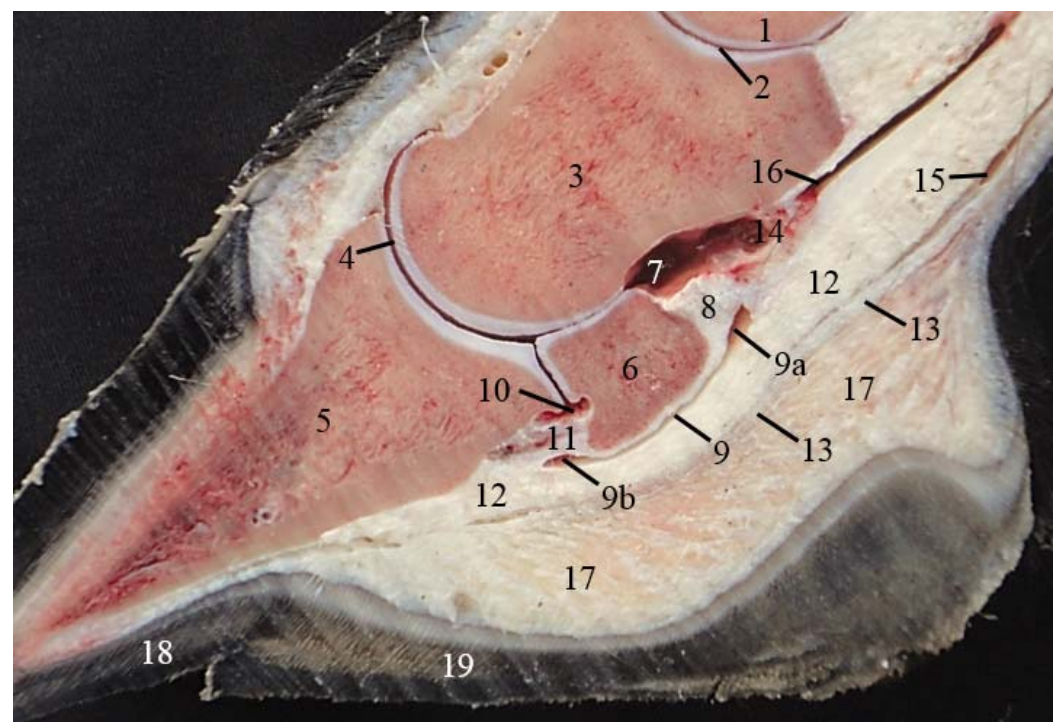

Figura 1. Aspecto macroscópico normal do aparato podotroclear de uma peça anatômica do membro torácico de um equino adulto. 1. falange proximal; 2. articulação interfalangeana proximal; 3. falange média; 4. articulação interfalangeana distal; 5. falange distal; 6. osso sesamoide distal (navicular); 7. recesso palmar proximal da articulação interfalangeana distal; 8. ligamento sesamoide colateral (suspensor); 9. bursa podotroclear; 9a. recesso proximal da bursa podotroclear; 9b. recesso distal da bursa podotroclear; 10. recesso palmar distal da articulação interfalangeana distal; 11. ligamento sesamoide distal ímpar; 12. tendão do músculo flexor digital profundo; 13. ligamento anular digital distal; 14. ramos arteriais palmares da falange média; 15. recesso palmar distal da bainha digital; 16. recesso dorsal distal da bainha digital; 17. coxim digital; 18. sola; 19. ranilha.

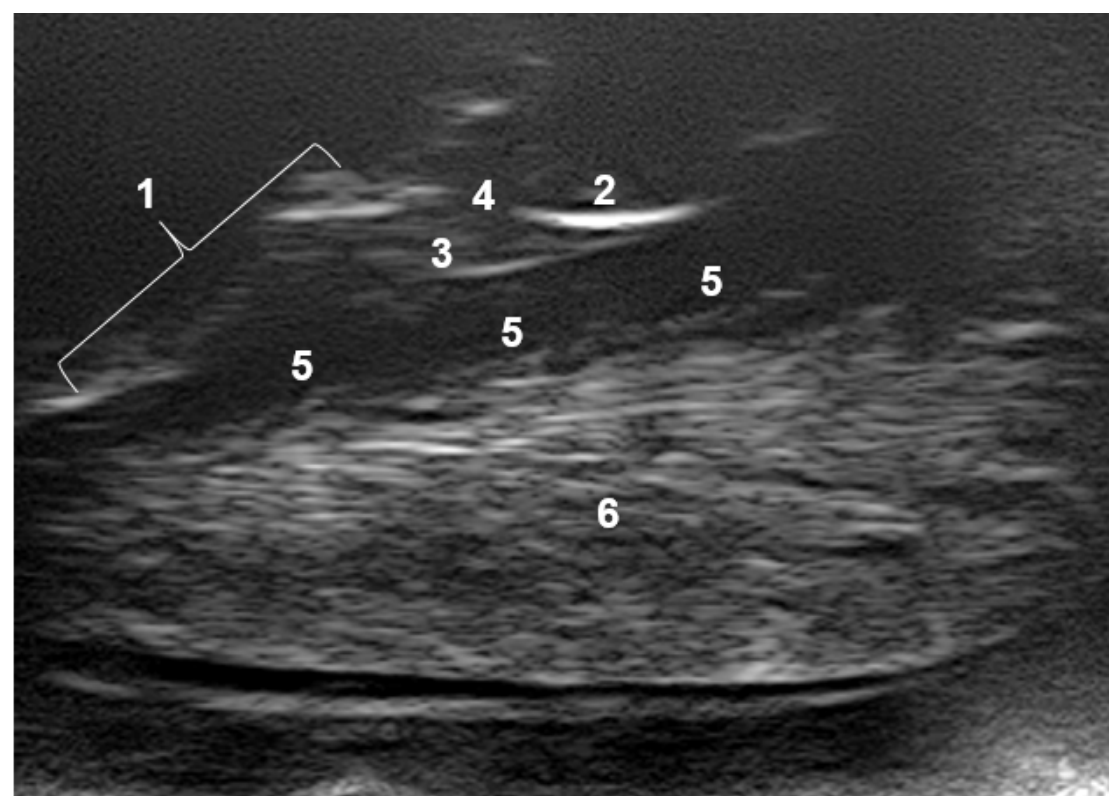

Figura 2. Imagem ultrassonográfica normal (plano sagital) pela técnica transcuneal da região palmar do casco, evidenciando o aparato podotroclear de uma peça do membro torácico de um equino adulto. 1. superfície óssea palmar da falange distal; 2. superfície óssea flexora do osso sesamoide distal (navicular); 3. ligamento sesamoide distal ímpar; 4. recesso palmar distal da articulação interfalangeana distal; 5. tendão do músculo flexor digital profundo/ligamento anular digital distal; 6. coxim digital. 


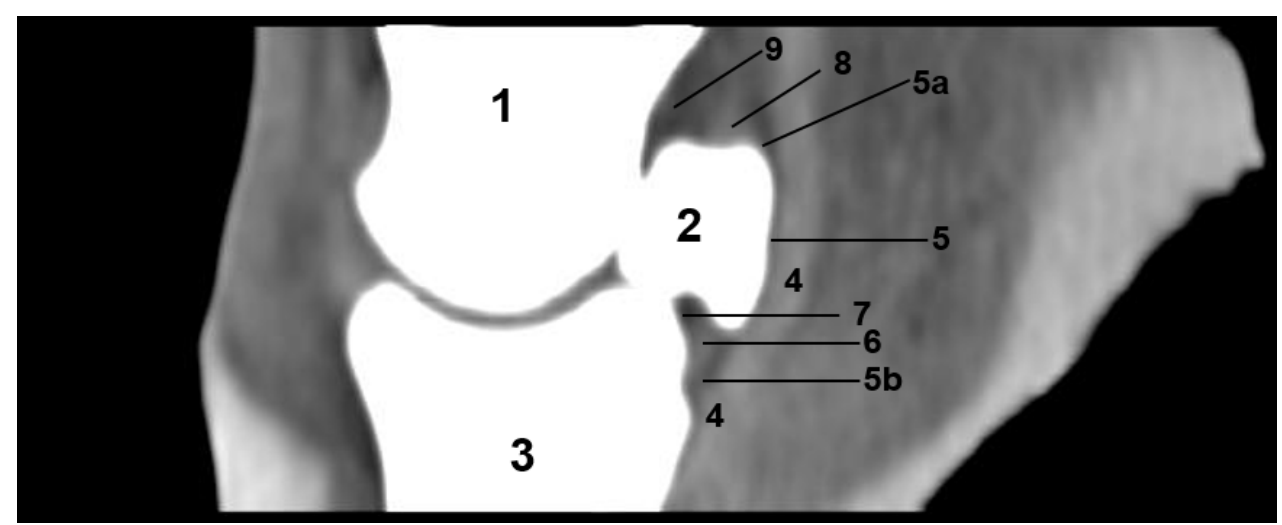

Figura 3. Imagem tomográfica normal no plano sagital (em janela para tecidos moles) do aparato podotroclear de uma peça do membro torácico de um equino adulto. 1. falange média; 2. osso sesamoide distal (navicular); 3. falange distal; 4. tendão do músculo flexor digital profundo/ligamento anular digital distal; 5. bursa podotroclear; 5a. recesso proximal da bursa podoroclear; 5b. recesso distal da bursa podotroclear; 6. ligamento sesamoide distal ímpar; 7. recesso palmar distal da articulação interfalangeana distal; 8. ligamento sesamoide colateral (suspensor); 9. recesso palmar proximal da articulação interfalangeana distal.

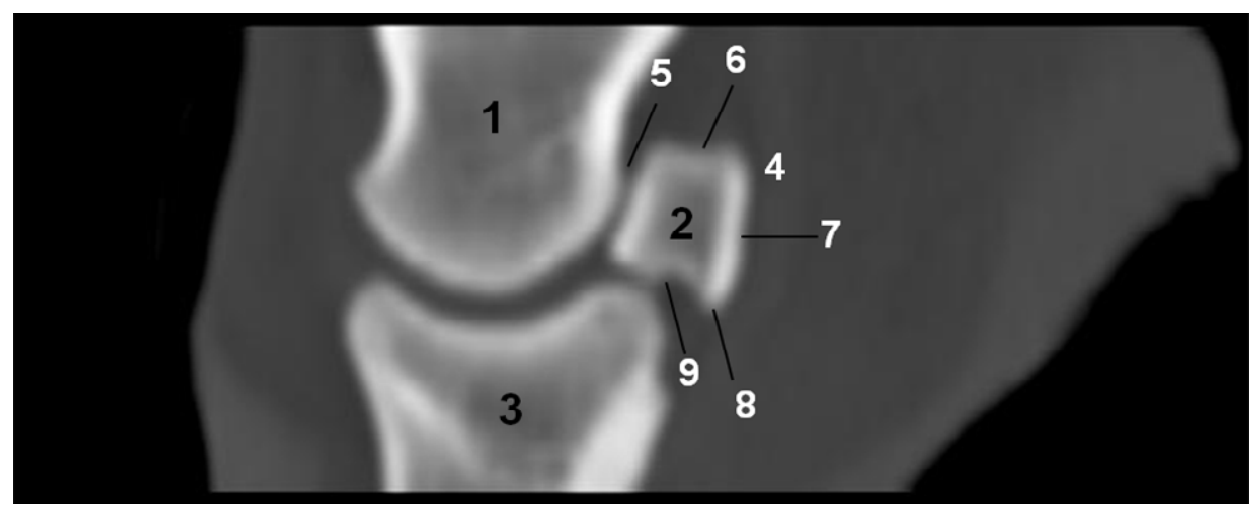

Figura 4. Imagem tomográfica normal no plano sagital (em janela óssea) do aparato podotroclear de uma peça do membro torácico de um equino adulto. 1. falange média; 2. osso sesamoide distal (navicular); 3. falange distal; 4. tendão do músculo flexor digital profundo 5. superfície articular do osso sesamoide distal; 6. borda proximal do osso sesamoide distal; 7. superfície flexora do osso sesamoide distal; 8. borda flexora distal do osso navicular; 9. borda distal do osso sesamoide distal.

Entre o osso navicular e o TFDP, forma-se uma bolsa sinovial, denominada bursa podotroclear, e suas evaginações são intituladas recesso proximal e recesso distal da bursa podotroclear. Imediatamente palmar à bursa, está o TFDP e, em seu contorno palmar, pode-se observar o LADD. Esse ligamento tem a função de sustentar a porção terminal do TFDP, separando-o do coxim digital. Os ramos arteriais palmares da falange média são vistos seccionados e proximais em relação ao ligamento sesamoide colateral. Distal e palmar, encontra-se o coxim digital, a sola e a ranilha.
No exame ultrassonográfico pela técnica transcuneal do aparato podotroclear, é possível avaliar a superfície óssea palmar da falange distal, a superfície óssea (flexora) do osso navicular, o LSDI e sua inserção na falange distal, o recesso palmar distal da articulação interfalangeana distal, o recesso distal da bursa podotroclear, o TFDP e o coxim digital (Fig. 2).

A superfície óssea palmar da falange distal foi evidenciada como uma suave linha hiperecoica regular e contínua, na qual se inserem, na porção mais proximal, o LSDI, e na porção mais distal, o TFDP. A superfície flexora do osso navicular é visibilizada como uma linha hiperecoica, 
localizada dorsal ao TFDP. Da borda distal dessa superfície, origina-se o LSDI. Este é um ligamento pequeno, que ocupa um espaço triangular entre o TFDP, a superfície hiperecoica da falange distal e a borda distal do osso navicular. Sua ecogenicidade é frequentemente maior quando comparada com a ecogenicidade do TFDP. O recesso palmar distal da articulação interfalangeana distal é visibilizado como uma estrutura anecoica dorsal ao LSDI.

O TFDP é visibilizado como uma banda de aparência hipoecoica a anecoica no aspecto palmar da superfície flexora do osso navicular, seguindo em sentido distal até sua inserção na face palmar da falange distal, não sendo possível a avaliação do padrão de suas fibras. A bursa podotroclear é localizada entre a superfície flexora do osso navicular e o TFDP e consiste em uma estrutura anecoica, a qual não pode ser diferenciada do TFDP. Dessa forma, a estrutura anecoica localizada palmar à superfície óssea (flexora) do osso navicular representa a superfície cartilaginosa do osso navicular, a bursa podotroclear e o TFDP. O LADD não é diferenciado do TFDP no seu aspecto palmar. $\mathrm{O}$ coxim digital consiste em uma estrutura heterogênea e hiperecoica, sendo a primeira estrutura visibilizada.

No corte sagital do exame tomográfico, em janela de tecidos moles (Fig. 3), foi visibilizada a falange média, o osso navicular e a falange distal como estruturas hiperatenuantes sem diferenciação entre cortical e medular. O TFDP e o LADD originam-se próximo à superfície palmar do sesamoide distal, estendendo-se além da falange média, e apresentam-se como uma estrutura filiforme, homogênea, de margens claramente demarcadas, com maior atenuação que os tecidos moles adjacentes.

A bursa podotroclear foi pouco visível, apresentando-se como uma pequena linha hipoatenuante entre a margem da superfície flexora do osso navicular e o TFDP. O recesso proximal da bursa podotroclear foi bem identificado como uma estrutura hipoatenuante, localizado proximal ao osso navicular. O recesso palmar distal da articulação interfalangeana distal apresenta-se hipoatenuante e bem delimitado em sua porção dorsal pela superfície óssea palmar da falange distal e pelo osso navicular, e, em sua porção palmar, pela presença do LSDI. O LSDI foi identificado como uma faixa homogênea e delgada quando comparado ao TFDP, tendo igual atenuação. Imediatamente palmar a este, visibilizou-se a porção distal do recesso distal da bursa podotroclear como uma estrutura hipoatenuante, sendo delimitada em sua porção palmar pelo TFDP.

O ligamento sesamoide colateral (suspensor) foi visto como uma estrutura ligeiramente triangular, hiperatenuante, que se estende a partir do aspecto proximal da superfície do osso navicular, delimitado dorsalmente pelo recesso palmar proximal da articulação interfalangeana distal e, no seu aspecto palmar, pelo recesso proximal da bursa podotroclear. Os recessos palmares proximal e distal da articulação interfalangeana distal são visibilizados como estruturas hipoatenuantes e homogêneas. $\mathrm{O}$ recesso palmar proximal é delimitado dorsalmente pela falange média e distalmente pelo osso navicular. Por sua vez, o recesso palmar distal da articulação interfalangeana distal apresenta relação proximal com o osso navicular e distal com a falange distal.

No corte sagital do exame de tomografia computadorizada, na avaliação em janela para o tecido ósseo (Fig. 4), pode-se visualizar, na face palmar entre a falange média e o osso navicular, uma fina faixa hipoatenuante, a qual corresponde à superfície articular. No aspecto proximal do osso navicular, pode-se visibilizar sua borda proximal; já na região palmar, encontra-se a superfície flexora e, na porção distal da superfície flexora, encontra-se a borda flexora distal. A borda distal do osso navicular localizase na região distal dele e possui contato com a falange distal. $\mathrm{Na}$ porção palmar a essas estruturas, é possível visualizar o TFDP, com aspecto homogêneo e levemente hiperatenuante quando comparado aos tecidos moles adjacentes, o que dificulta sua avaliação.

\section{DISCUSSÃO}

O presente trabalho não descreve apenas a anatomia podotroclear de equinos hígidos por meio da avaliação e descrição de estruturas específicas em peças anatômicas, mas também a avaliação qualitativa das estruturas por exames de imagem, incluindo a ultrassonografia na técnica transcuneal e a tomografia 
computadorizada. Em todas as peças anatômicas, sob avaliação macroscópica, as delimitações do TFDP foram claramente demarcadas, e não foram observadas alterações no osso navicular e nas outras estruturas do aparato podotroclear.

$\mathrm{Na}$ avaliação ultrassonográfica, as estruturas visualizadas foram: LSDI e TFDP, incluindo suas inserções na borda palmar da falange distal; recesso palmar distal da articulação interfalangeana distal; superfície óssea (flexora) do osso navicular e coxim digital (Busoni e Denoix, 2001; Sage e Turner, 2002; Busoni et al., 2006; Whitcomb, 2009). A avaliação ultrassonográfica das estruturas proximais ao osso navicular é prejudicada devido à sombra acústica da sua superfície óssea.

A avaliação do TFDP é limitada devido à impossibilidade de se visualizar o seu padrão de fibras, o que está de acordo com o observado por Busoni et al. (2006) e Whitcomb (2009). Segundo Whitcomb (2009), em alguns equinos, o padrão de fibras do TFDP pode ser visibilizado no nível do LSDI e na inserção na falange distal, no entanto tal padrão não foi observado em nenhuma das imagens realizadas no presente estudo. De acordo com Busoni e Denoix (2001), apenas quando o feixe de ultrassom é perpendicular às fibras do tendão, este aparece ecogênico, e a interface entre o coxim digital, o LADD e o TFDP, torna-se visível. Em estudo realizado por Busoni et al. (2006), o diagnóstico de lesão no TFDP foi realizado pela avaliação do contorno palmar do tendão, no qual a convexidade anormal ou a ondulação palmar foi associada à lesão.

A bursa podotroclear não deve ser confundida com a estrutura anecoica localizada palmar à superfície flexora do osso navicular, a qual se refere ao conjunto das seguintes estruturas: superfície cartilaginosa do osso navicular, bursa podotroclear e TFDP (Whitcomb, 2009).

A avaliação ultrassonográfica da superfície óssea (flexora) do osso navicular é possibilitada pela técnica transcuneal, sendo essencial, já que defeitos nessa superfície podem ser identificados por meio da ultrassonografia (Whitcomb, 2009). De acordo com Grassi et al. (2001), a ultrassonografia é um exame de alta sensibilidade na detecção de irregularidades na superfície óssea e pode confirmar achados radiológicos equivocados ou auxiliar na identificação de alterações precoces do córtex flexor do osso navicular. Além disso, a projeção palmaroproximal-palmarodistal obliqua (skyline) do osso navicular pode ser de difícil realização em equinos portadores de dor palmar, devido à extensão da articulação interfalangeana distal induzida durante o exame. Dessa forma, o exame ultrassonográfico oferece uma alternativa viável para investigação do córtex flexor do osso navicular (Busoni et al., 2006).

Todas as estruturas observadas nas peças anatômicas foram visibilizadas na tomografia computadorizada, com exceção dos ramos arteriais palmares da falange média. Neste estudo, não foi possível a diferenciação ultrassonográfica e a tomográfica entre o TFDP e o LADD, que, em condições normais, apresentam similaridade na ecogenicidade e atenuação das fibras, o que corrobora o estudo realizado por Busoni et al. (2006). Segundo Grewal et al. (2004), o LADD, algumas vezes, pode parecer mais hiperecogênico que o TFDP. A mensuração dessas estruturas é realizada em conjunto devido à dificuldade de se delinearem as margens do TFDP das margens do LADD (Grewal et al., 2004).

Relatos referentes ao uso da tomografia computadorizada para avaliação da extremidade distal de equinos são limitados. De acordo com Whitton et al. (1998) e Widmer et al. (2000), a tomografia computadorizada tem sido relatada como um método de diagnóstico que fornece excelentes detalhes na avaliação óssea, porém é pobre na delineação de tecidos moles. Em contrapartida, este estudo demonstra que a avaliação de tecidos moles do aparato podotroclear pode ser realizada quando forem utilizados janela própria e nivelamento apropriados.

A introdução do meio de contraste arterial pode melhorar a anatomia e a fisiologia subjacente por meio da tomografia computadorizada, o que aumenta a utilidade dessa modalidade de imagem para o diagnóstico de lesões nos tecidos moles (Puchalski et al., 2007). Apesar de não ter sido possível realizar o exame com contraste arterial neste estudo, a tomografia computadorizada forneceu bom detalhamento anatômico. 


\section{CONCLUSÃO}

Conclui-se que o exame ultrassonográfico pela técnica transcuneal permite adequada avaliação das estruturas palmares do casco e pode ser utilizado na rotina clínica como exame complementar para o diagnóstico de claudicações relacionadas a lesões no aparato podotroclear. O exame tomográfico fornece imagens com melhor riqueza de detalhes da cortical e do trabeculado dos elementos ósseos que compõem o aparato podotroclear, além da possibilidade de visibilizar e avaliar estruturas de tecido moles em janela específica.

\section{REFERÊNCIAS}

BUDRAS, K.D.; KÖNIG, H.E. Casco (úngula) do cavalo. In: KÖNIG, H.E.; LIEBICH, H.G. (Eds.). Anatomia dos animais domésticos: texto e atlas colorido. Porto Alegre: Artmed, 2004. p.264-374.

BUSONI, V.; DENOIX, J.M. Ultrasonography of the podotrochlear apparatus in the horse using a transcuneal approach: technique and reference images. Vet. Radiol. Ultrasound, v.42, p.534540, 2001.

BUSONI, V.; LAHAYE, B.; DENOIX, J.M. Transcuneal ultrasonographic findings in the podotrochlear apparatus: comparison with postmortem in 14 equine digits. J. Equine Vet. Sci., v.26, p.113-119, 2006.

CLAYTON, H.M.; FLOOD, P.F. (Eds.). Atlas colorido de anatomia aplicada dos grandes animais. 3.ed. Barueri: Manole, 2002. p.54-63.

DENOIX, J.M. (Ed.). The equine distal limb: an atlas of clinical anatomy and comparative imaging. London: Manson, 2000. 390p.

DYSON, S.; MARKS, D. Foot pain and the elusive diagnosis. Vet. Clin. N. Am. Equine Pract., v.19, p.531-565, 2003.

FIO, L.; KOBLIK, P.D. Computed axial tomography (CAT or CT scanning) - veterinary review. J. Equine Vet. Sci., v.15, p.511-513, 1995.

GRASSI, W.; FILIPPUCCI, E.; FARINA, A. et al. Ultrasonography in the evaluation of bone erosions. Ann. Rheum. Dis., v.60, p.98-103, 2001.
GREWAL, J.S.; McCLURE, S.R.; BOOTH, L.C. et al. Assessment of the ultrasonographic characteristics of the podotrochlear apparatus in clinically normal horses and horses with navicular syndrome. J. Am. Vet. Med. Assoc., v.225, p.1881-1888, 2004.

POPESKO, P. (Ed.). Atlas de anatomia topográfica dos animais domésticos. São Paulo: Manole, 1997. v.3, 205p.

PUCHALSKI, S.M.; GALUPPO, L.D.; HORNOF, W.J.; WISNER, E.R. Intraarterial contrast-enhanced computed tomography of the equine distal extremity. Vet. Radiol. Ultrasound, v.48, p.21-29, 2007.

RIJKENHUIZEN, A.B.M. Navicular disease: a review of what's new. Equine Vet. J., v.38, p.8288, 2006.

SAGE, A.M.; TURNER, T.A. Ultrasonography of the soft tissue structures of the equine foot. Equine Vet. Educ., v.14, p.221-224, 2002.

STASHAK, T.S. Claudicação In: Claudicação em equinos segundo Adams. 5.ed. São Paulo: Roca, 2005. p.517-532.

TURNER, A.T. Diagnosis and treatment of the navicular syndrome in horses. Vet. Clin. N. Am. Equine Pract., v.5, p.131-144, 1989.

WHITCOMB, M.B. Ultrasonographic evaluation of the distal extremity. J. Equine Vet. Sci., v.29, p.47-59, 2009.

WHITTON, R.C.; BUCKLEY, C.; DONOVAN, T. et al. The diagnosis of lameness associated with distal limb pathology in a horse: a comparison of radiography, computed tomography and magnetic resonance imaging. Vet. J., v.155, p.223-229, 1998.

WIDMER, W.R.; BUCKWALTER, K.A.; FESSLER, J.F. et al. Use of radiography, computed tomography and magnetic resonance imaging for evaluation of navicular syndrome in the horse. Vet. Radiol. Ultrasound, v.41, p.108$116,2000$.

WRIGHT, I.M. A study of 118 cases of navicular disease: radiological features. Equine Vet. J., v.25, p.493-500, 1993. 